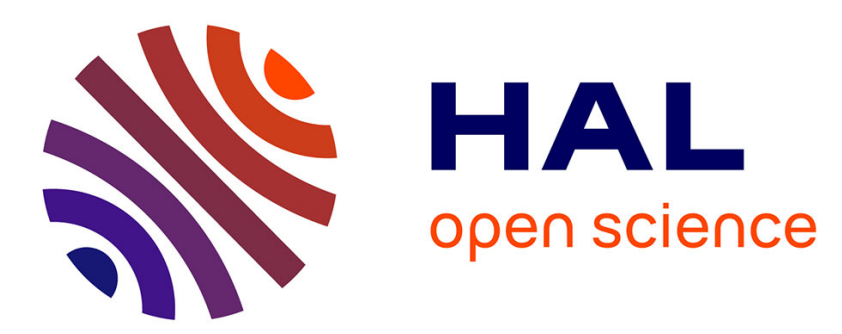

\title{
Emergence of extreme events in fiber-based parametric processes driven by a partially incoherent pump wave
}

Kamal Hammani, Christophe Finot, Guy Millot

\section{To cite this version:}

Kamal Hammani, Christophe Finot, Guy Millot. Emergence of extreme events in fiber-based parametric processes driven by a partially incoherent pump wave. Optics Letters, 2009, 34 (8), pp.1138-1140. 10.1364/OL.34.001138 . hal-00408626

\section{HAL Id: hal-00408626 https://hal.science/hal-00408626}

Submitted on 20 Apr 2010

HAL is a multi-disciplinary open access archive for the deposit and dissemination of scientific research documents, whether they are published or not. The documents may come from teaching and research institutions in France or abroad, or from public or private research centers.
L'archive ouverte pluridisciplinaire HAL, est destinée au dépôt et à la diffusion de documents scientifiques de niveau recherche, publiés ou non, émanant des établissements d'enseignement et de recherche français ou étrangers, des laboratoires publics ou privés. 


\title{
Emergence of extreme events in fiber-based parametric
}

\section{processes driven by a partially incoherent pump wave}

\author{
Kamal Hammani, Christophe Finot, ${ }^{*}$ and Guy Millot \\ Institut Carnot de Bourgogne, UMR 5209 CNRS/Université de Bourgogne, 21078 Dijon, France \\ *Corresponding author: christophe.finot@u-bourgogne.fr
}

We present experimental and theoretical results showing efficient emergence of rogue wave-like extreme intensity spikes during the fiber-based induced-modulational instability process driven by a partially incoherent pump. In particular, we show that the rogue event probability can be easily controlled by adjusting the pump-signal detuning.

OCIS codes: 060.4370 (Nonlinear optics, fibers), 060.7140 (Ultrafast processes in fibers), 060.2320 (Fiber optics amplifiers and oscillators), 190.4410 (Nonlinear optics, parametric processes), 190.3100 (Instabilities and chaos). 
Optical fiber systems are well-known to provide convenient platforms with which to investigate a large and diverse set of fascinating fundamental nonlinear phenomena [1]. Among the striking phenomena due to the interplay of the dispersive and Kerr effects is the modulational instability (MI) [2], entailing the exponential growth of a weak perturbation at the expense of a continuous or quasi-continuous pump wave. The amplified perturbation can be either quantum noise (i.e., spontaneous MI) or a frequency shifted signal wave (i.e., induced MI). In the frequency domain, MI is equivalent to a four-photon parametric mixing process where two pump photons are annihilated to create a Stokes (signal) - anti-Stokes (idler) photon pair. For both its fundamental and applied interests, MI has attracted a great attention more than twenty years ago. In view of its potential application to fiber-based communication systems, several MI-based devices were proposed such as wide-band optical parametric amplifiers, wavelength converters or highrepetition rate lasers $[1,3-5]$.

More recently, MI generated by a picosecond coherent pulse has been shown to be the key ingredient of the generation of optical rogue-waves in supercontinuum $[6,7]$ : by analogy with hydrodynamics and the Benjamin-Feir instability [8], Solli et al. have highlighted that MI could explain the emergence of such rare but intense optical events. These extreme statistics, which deviate significantly from Gaussian probability, can also appear in non-solitonic systems such as Raman amplifiers driven by a partially incoherent pump wave propagating in fibers with low group velocity dispersion [9]. In such a system, the initial intensity fluctuations of the pump are indeed exponentially amplified, resulting in the generation of intense spikes with temporal duration corresponding to the pump coherence time.

The question which we address here is concerned with the impact of a partially incoherent pump on the performances of parametric (MI) amplifiers. Indeed, since both Raman 
and parametric amplifiers are based on a fast amplification process which follows the rapid optical field fluctuations inherent to an incoherent wave, one may naturally wonder whether optical rogue events can also occur in MI-based amplifier. In preliminary investigations, Sylvestre et al. have outlined that partially incoherent pumping, produced by fiber Raman lasers, could significantly deteriorate the quality of the amplified signals [10]. However, their analysis has been restricted to the frequency domain so that, to the best of our knowledge, the temporal profile and the associated statistical properties of an amplified signal have not been yet investigated in detail. In this Letter, we provide experimental evidence of rare optical events in a parametric amplifier in presence of a strong partially incoherent pump. We stress, both experimentally and numerically, the fundamental differences between MI with coherent and partially incoherent pumping schemes. On the other hand, we describe the temporal evolution experienced by a continuous signal and its variation as a function of the pump-signal frequency detuning. Finally, the use of a pulsed signal offers the possibility to carry out more precise measurements on the pulse statistics and clearly highlights the impact of the incoherent pumping.

Our all-fibered experimental setup is sketched on Fig. 1(a) and relies exclusively on commercially available telecommunication-ready components. We have designed a simple home-made partially incoherent source by amplifying the spectrally sliced and polarized amplified spontaneous emission (ASE) of an erbium-doped fiber amplifier (EDFA). Spectral slicing of the ASE emerging from a first EDFA was achieved by means of an optical bandpass filter (OBPF) having a Gaussian profile with a spectral full-width at half-maximum (FWHM) of $40 \mathrm{GHz}$ (Fig. 1(b)). An additional OBPF is included in order to remove potential ASE generated by a second EDFA used to amplify the filtered ASE of the first EDFA. This system enabled us to 
reach an optical signal to noise ratio higher than $50 \mathrm{~dB}$ for a maximum average power of 110 $\mathrm{mW}$ (the output power is controlled using a variable optical attenuator (OA) ). The temporal properties of this incoherent pump have been analyzed through autocorrelation measurements (Fig. 1(c)). Experimental results are in good agreement with the assumption of stochastic fluctuations with a Gaussian probability distribution [9]. Characteristics time of the fluctuations has then estimated to be $9 \mathrm{ps,}$, in agreement with the spectral measured bandwidth.

The partially incoherent pump and a continuous signal with an average power of $140 \mu \mathrm{W}$ were combined into a 500-m highly nonlinear fiber (HNLF) with a nonlinear coefficient $\gamma$ of $10 \mathrm{~W}^{-1} \cdot \mathrm{km}^{-1}$ and a second order dispersion $\beta_{2}=-6 \times 10^{-4} \mathrm{ps}^{2} \cdot \mathrm{m}^{-1}$. We have first recorded the output spectra for various pump-signal detunings $\Omega=\left|v_{s}-v_{p}\right|$ ranging from 0.1 to $1.8 \mathrm{THz}$ (with $v_{s}$ and $v_{p}$ the central frequencies of the signal and pump, respectively). Results are summarized in Fig. 2(a1) and confirm the generation of an idler wave (up to $\Omega=1.2 \mathrm{THz}$ ) as well as significant cascading of the process (between $\Omega=0.4$ and $0.8 \mathrm{THz}$ ). We have carefully compared these results with those obtained with a coherent pump delivered by an external cavity laser (Fig. 2(a2)). Major differences are then clearly outlined: visible idler is generated only up to $0.8 \mathrm{THz}$ and no cascading is observed. We can then conclude that the amplification bandwidth generated by a partially incoherent pump is broader than the one generated by a coherent pump. On the other hand, for similar average pumping powers, the resulting MI gain is higher with a partially incoherent pump. Both results are fully consistent with a previous analytical and experimental study carried out in spontaneous partially incoherent MI [11]. Let us also note that the resulting spectral bandwidth of the amplified signal is significantly increased in presence of incoherent pumping, due to the cross-phase modulation of the pump on the signal [12]. In order 
to validate our experimental approach, we have simulated the longitudinal evolution of the envelope of the complex electric field $\psi(\mathrm{z}, \mathrm{T})$ through the standard nonlinear Schrödinger (NLS) equation [1] :

$$
i \frac{\partial \psi}{\partial z}=\frac{\beta_{2}}{2} \frac{\partial^{2} \psi}{\partial T^{2}}-\gamma|\psi|^{2} \psi
$$

Numerical integration of this equation by the usual split-step Fourier method (Figs. 2(b)) exhibits an excellent quantitative agreement with the experimental results.

We then got interested in the temporal evolution of the signal which is isolated from the pump and idler waves by means of an adequate OBPF (supergaussian shape, $1.25 \mathrm{THz}$ spectral FWHM). Temporal measurements recorded by a photodiode connected to a GHz-oscilloscope (Figure 3(a)) highlight the emergence of intense spikes on the amplified signal for various pumpsignal detunings. Changing the pump-signal detuning significantly impacts the number and intensity of the spikes, higher detuning leading to less frequent spikes (Fig. 3(a3)). Experimental results are in agreement with numerical simulations, as shown by Fig. 3(b) in the particular case of the largest frequency detuning (corresponding to Fig. 3(a3)). Therefore, the control of the pump-signal detuning represents a convenient way to directly influence the emergence probability of the rogue structures. Moreover, we have checked that such spiky structures are only observed with the partially incoherent pump. In order to get further insights on the fine temporal structure of the spikes [9], we have recorded the autocorrelation of the amplified signal. Results plotted on Fig. 3(c) highlight a temporal width of 5 ps. This temporal duration is of the same order of magnitude that the pump fluctuations presented on Fig. 1(c). However, let us note that this duration is nearly a factor 2 below the pump coherence time, which can be explained by the exponential gain experienced by the localized structure of the signal. The experimental 
amplified signal contrast is well below the initial contrast 1:2 of the pump, in agreement with the rogue nature of the amplified signal [9]. Such an evolution is well reproduced by numerical integration of the NLS equation.

In order to carry out quantitative statistical measurements, we have then substituted the continuous signal by a picosecond signal delivered by a mode-locked fiber laser running at a repetition rate of $22 \mathrm{MHz}$. As the pump-signal detuning is fixed to $1 \mathrm{THz}$, most of the pulse train does not experience any amplification as illustrated on Fig. 4(a): only rare pulses are amplified and therefore deviate strongly from the average output power level. Related experimental statistics, as recorded on a 50-GHz sampling oscilloscope, are plotted on Fig. 4(b1) for various pump powers. As the partially incoherent wave power increases, the probability distributions become clearly asymmetric and deviate from a purely Gaussian shape with a tail developing towards higher peak-powers. This evolution is qualitatively well reproduced by an extensive set of numerical simulations ( $10^{5}$ simulations) as shown in Fig. 4(b2). A quantitative difference may appear however due to the electronic noise of the detection system (photodiode and oscilloscope) which broadens the experimental distribution. We have not included this noise in our simulations but we have experimentally carefully checked that its influence does not depend on the pump power, contrary to the observed power-dependent wing broadening.

In conclusion, we have shown that induced-MI (or parametric amplification) based on a partially incoherent pump can lead to the emergence of high signal peaks deviating significantly from the average dynamics. Similarly to Raman amplification, this behavior can be explained by the instantaneous exponential transfer of the fluctuations of the pump to the signal. The rogue event probability can be easily controlled by adjusting the pump-signal detuning. By providing a new 
vision of the temporal evolution of a wave, this study may find potential applications in the design of parametric-based devices for optical communications.

We would like to thank S. Pitois, A. Picozzi and J.-M. Dudley for illuminating discussions. This work was supported by the Agence Nationale de la Recherche (ANR SUPERCODE, SOFICARS and MANUREVA projects: ANR-06-BLAN-0401-01, ANR-07-RIB-013-03 and ANR-08-SYSC-019, respectively) and by the Conseil Régional de Bourgogne. 
References including full titles :

1. G. P. Agrawal, Nonlinear Fiber Optics, Third Edition (Academic Press, San Francisco, CA, 2001).

2. K. Tai, A. Hasegawa, and A. Tomita, "Observation of modulational instability in optical fibers," Phys. Rev. Lett. 56, 135-138 (1986).

3. R. H. Stolen and J. E. Bjorkholm, "Parametric amplification and frequency conversion in optical fibers," IEEE J. Quantum Electron. 18, 1062-1072 (1982).

4. S. Coen and M. Haelterman, "Continuous-wave ultrahigh-repetition-rate pulse train generation through modulational instability in a passive fiber cavity," Opt. Lett. 26, 39-41 (2001).

5. M. E. Marhic, N. Kagi, T.-K. Chiang, and L. G. Kazovsky, "Broadband fiber optical parametric amplifiers," Opt. Lett. 21, 573-575 (1996).

6. D. R. Solli, C. Ropers, P. Koonath, and B. Jalali, "Optical rogue waves," Nature 450, 1054 (2007).

7. J. M. Dudley, G. Genty, and B. J. Eggleton, "Harnessing and control of optical rogue waves in supercontinuum generation " Opt. Express 16, 3644-3651 (2008).

8. A. Dyachenko and V. E. Zakharov, "On the formation of freak waves on the surface of deep water," JETP Letters 88, 307-311 (2008).

9. K. Hammani, C. Finot, J. M. Dudley, and G. Millot, "Optical rogue-wave fluctuations in fiber Raman amplifiers," Opt. Express 16, 16467-16474 (2008).

10. T. Sylvestre, A. Mussot, E. Lantz, and H. Maillote, "Impact of pump quality on the performances of fibre optical parametric amplifiers," in LEOS Winter Topical Meeting, 49-50 (2008).

11. A. Sauter, S. Pitois, G. Millot, and A. Picozzi, "Incoherent modulation instability in instantaneous nonlinear Kerr media," Opt. Lett. 30, 2143-2145 (2005).

12. J. T. Manassah, "Induced phase modulation by noisy fields," Opt. Lett. 16, 1379-1381 (1991). 
References without titles :

1. G. P. Agrawal, Nonlinear Fiber Optics, Third Edition (Academic Press, San Francisco, CA, 2001).

2. K. Tai, A. Hasegawa, and A. Tomita, Phys. Rev. Lett. 56, 135-138 (1986).

3. R. H. Stolen and J. E. Bjorkholm, IEEE J. Quantum Electron. 18, 1062-1072 (1982).

4. S. Coen and M. Haelterman, Opt. Lett. 26, 39-41 (2001).

5. M. E. Marhic, N. Kagi, T.-K. Chiang, and L. G. Kazovsky, Opt. Lett. 21, 573-575 (1996).

6. D. R. Solli, C. Ropers, P. Koonath, and B. Jalali, Nature 450, 1054 (2007).

7. J. M. Dudley, G. Genty, and B. J. Eggleton, Opt. Express 16, 3644-3651 (2008).

8. A. Dyachenko and V. E. Zakharov, JETP Letters 88, 307-311 (2008).

9. K. Hammani, C. Finot, J. M. Dudley, and G. Millot, Opt. Express 16, 16467-16474 (2008).

10. T. Sylvestre, A. Mussot, E. Lantz, and H. Maillote, in LEOS Winter Topical Meeting, 4950 (2008).

11. A. Sauter, S. Pitois, G. Millot, and A. Picozzi, Opt. Lett. 30, 2143-2145 (2005).

12. J. T. Manassah, Opt. Lett. 16, 1379-1381 (1991). 
Figure caption :

Fig. 1 : (a) Experimental setup for induced incoherent MI. (b) Pump optical spectrum. (c) Pump autocorrelation. The experimental measurements (solid black line) are compared with the results calculated numerically assuming a Gaussian stochastic distribution with a coherence time of 9 ps (grey circles).

Fig. 2 : Evolution of the output spectrum as a function of pump-signal detuning. Experimental results (subplots a) are compared with numerical results (subplots b). The evolution based on a partially incoherent pump (subplots 1) is compared with that observed with a coherent pump (subplots 2). Intensities are normalized with respect to the initial signal power.

Fig. 3 : (a1-a3) Amplified signal for various pump-signal detunings $\Omega$ : $0.75 \mathrm{THz}, 1 \mathrm{THz}$ and 1.25 THz (supblots a1, a2 and a3, respectively, corresponding to spectra A, B and C of Fig. 2(a1) ). Intensity is normalized with respect to the median value. (b) Simulation of the amplified signal generated for a pump-signal detuning of $1.25 \mathrm{THz}$. (c) Experimental autocorrelation of the amplified signal (solid black line) compared with simulation results (grey line).

Fig. 4 : (a) Output pulsed signal in presence of a partially incoherent pump. The dashed line represents the signal power level without pump. (b) Probability distributions of the output peakpower for pump powers of $10 \mathrm{~mW}, 50 \mathrm{~mW}$ and $100 \mathrm{~mW}$ (light grey, dark grey and black histograms, respectively). Experimental results (b1) are normalized with respect to the median value and are compared with numerical results (b2). 

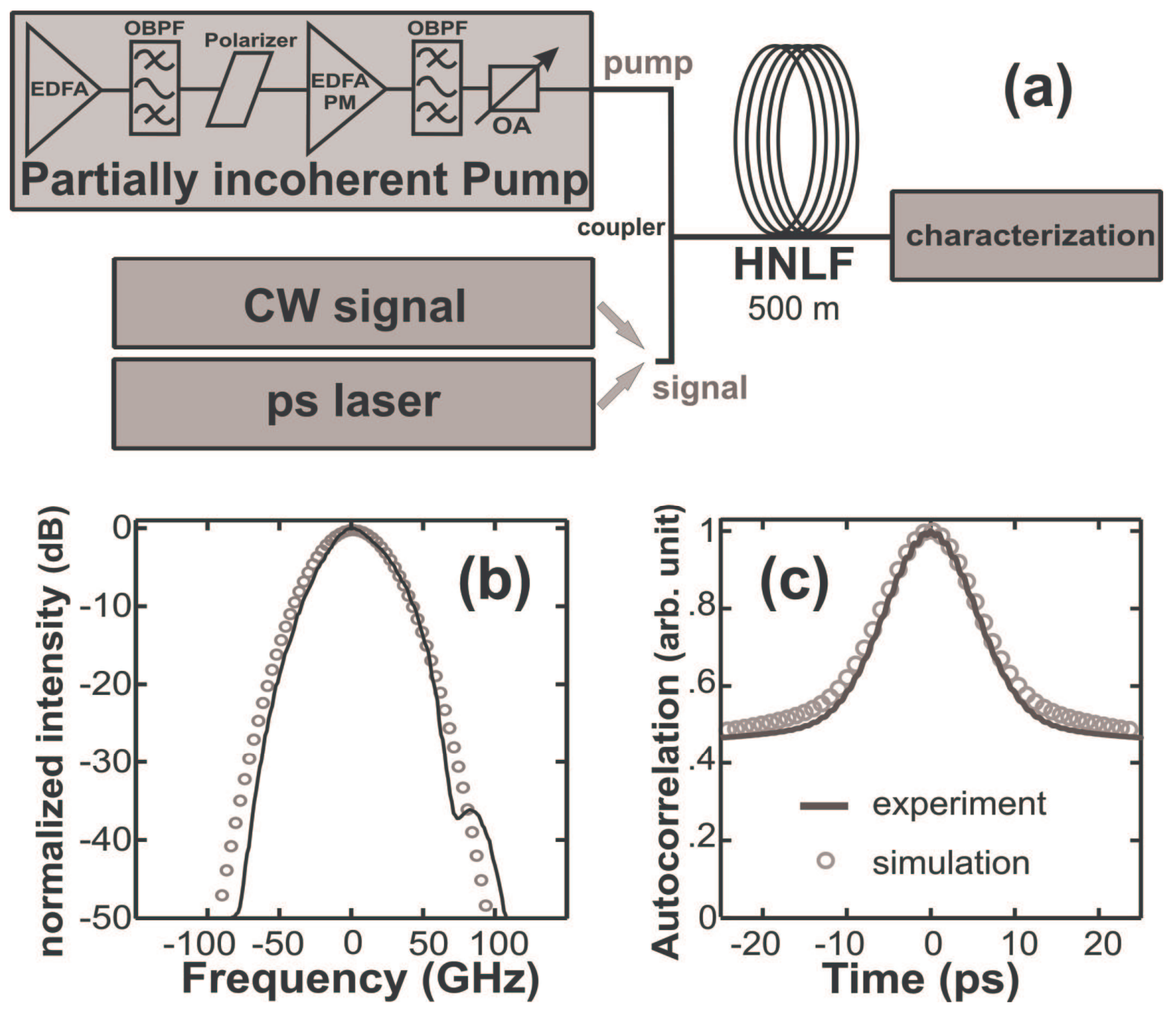

Figure 1 


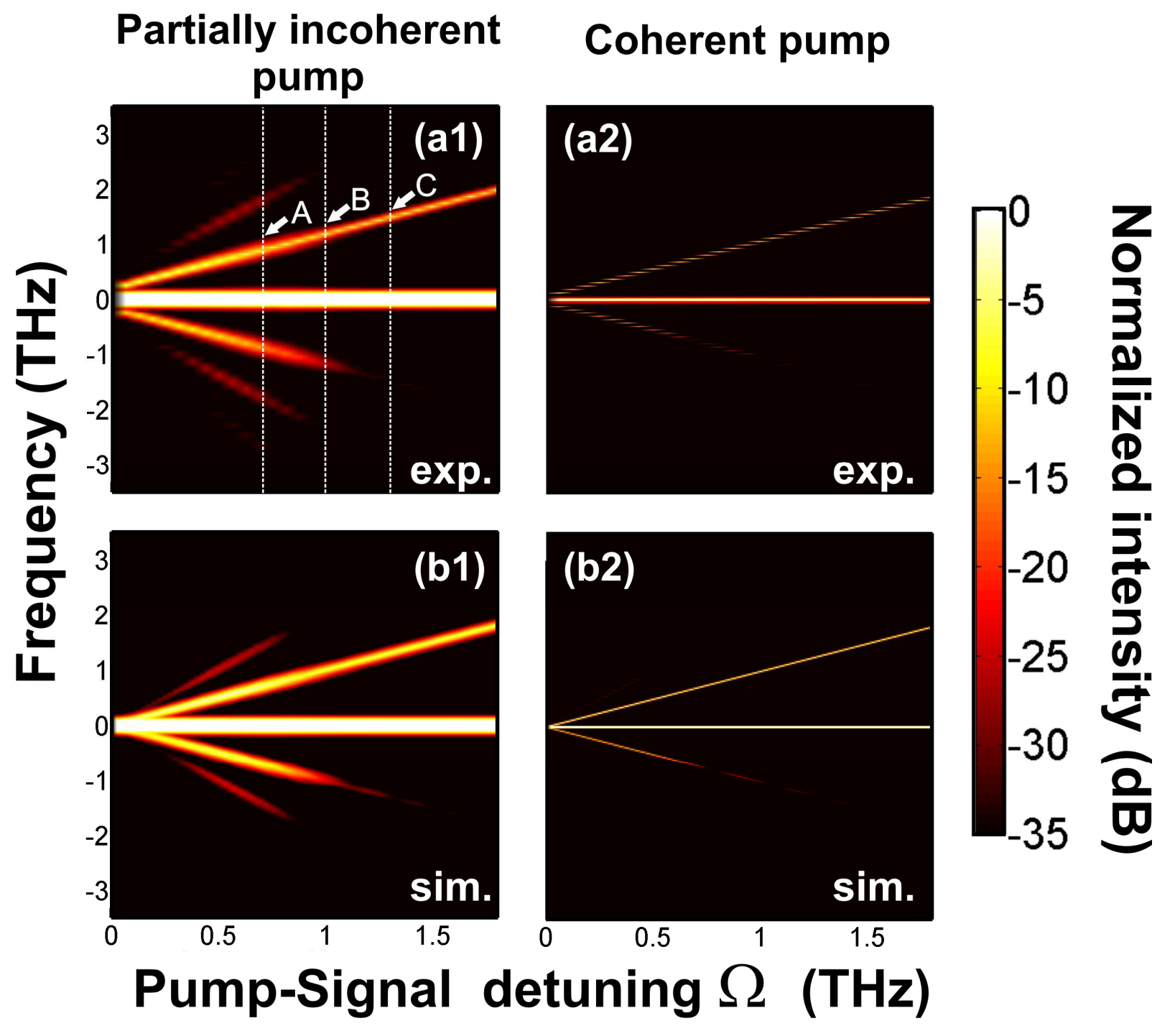

Figure 2 

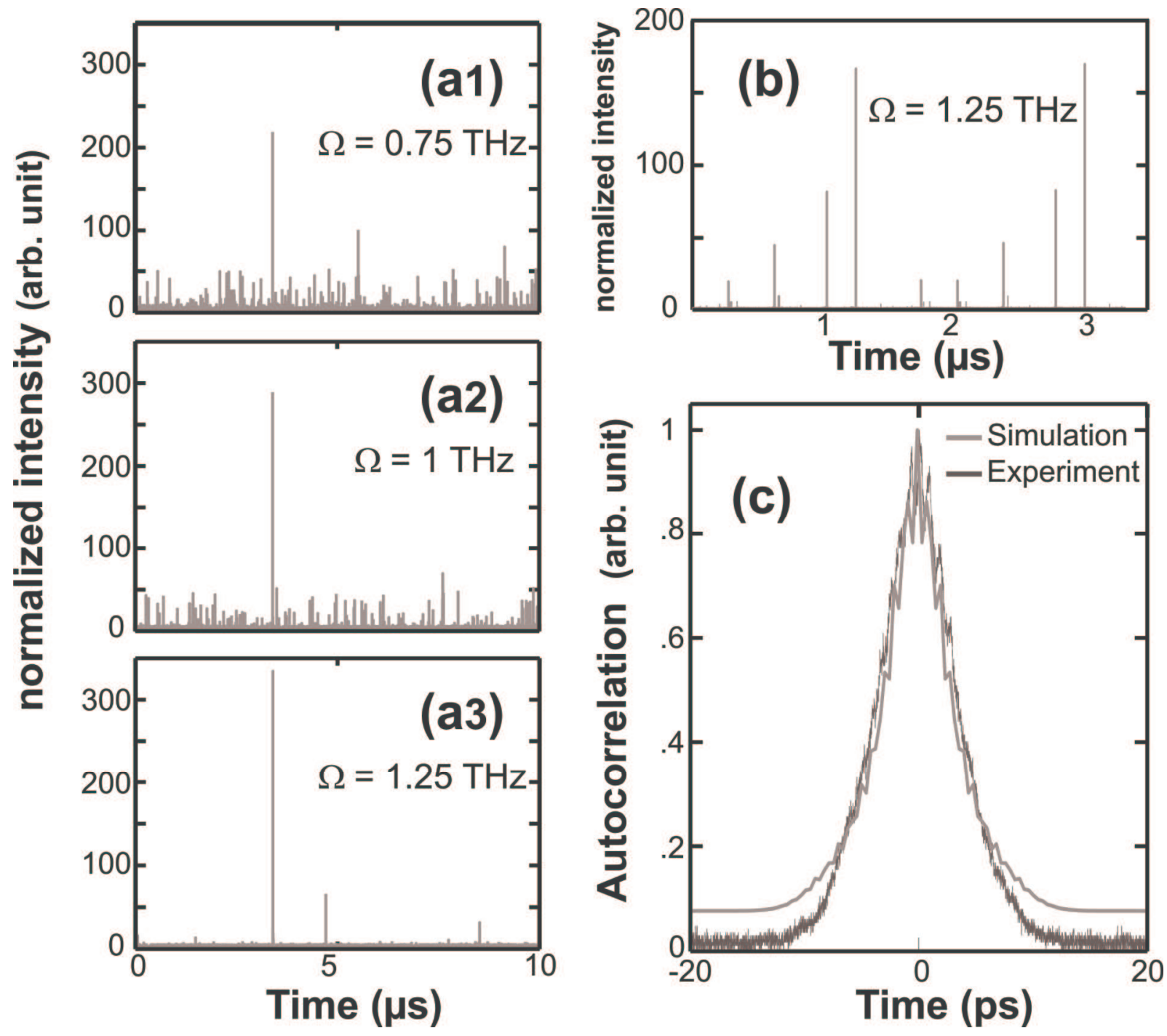

Figure 3 

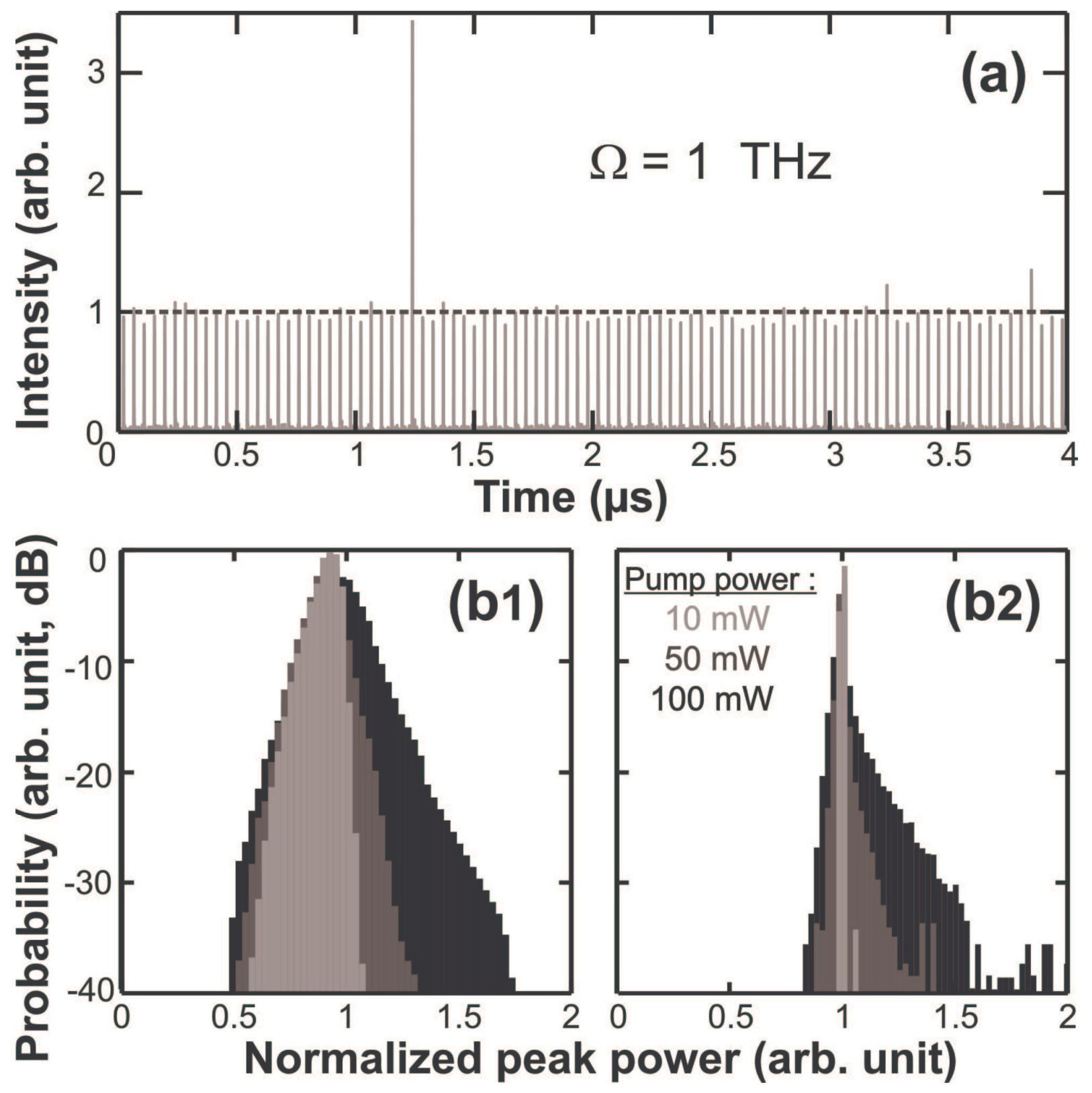

Figure 4 